TRANSACTIONS OF THE

AMERICAN MATHEMATICAL SOCIETY

Volume 358, Number 7, Pages 3097-3112

S 0002-9947(05)03794-3

Article electronically published on October 31,2005

\title{
A STRUCTURE THEOREM FOR THE ELEMENTARY UNIMODULAR VECTOR GROUP
}

\author{
SELBY JOSE AND RAVI A. RAO
}

\begin{abstract}
Given a pair of vectors $v, w \in R^{r+1}$ with $\langle v, w\rangle=v \cdot w^{T}=$ 1 , A. Suslin constructed a matrix $S_{r}(v, w) \in S l_{2^{r}}(R)$. We study the subgroup $S U m_{r}(R)$ generated by these matrices, and its (elementary) subgroup $E U m_{r}(R)$ generated by the matrices $S_{r}\left(e_{1} \varepsilon, e_{1} \varepsilon^{T^{-1}}\right)$, for $\varepsilon \in E_{r+1}(R)$. The basic calculus for $\operatorname{EUm}_{r}(R)$ is developed via a key lemma, and a fundamental property of Suslin matrices is derived.
\end{abstract}

\section{INTRODUCTION}

In his doctoral thesis A. Suslin showed that a unimodular vector of the form $\left(a_{0}, a_{1}, a_{2}^{2}, \cdots, a_{r}^{r}\right)$ can be completed to an invertible matrix (cf. [4, Proposition 1.6]). In the beautiful and thought provoking section $\S 5$ of this paper he gives an inductive (on $r$ ) method of constructing the completion. Given a pair of vectors $v, w \in M_{1 r+1}(R)$ he defines a matrix $S_{r}(v, w) \in M_{2^{r}}(R)$, with determinant equal to the inner product $v \cdot w^{T}$. Consequently, if the dot product is 1 , then $S_{r}(v, w) \in$ $S l_{2^{r}}(R)$. The actual completion is shown to be in the class $S_{r}(v, w) E_{2^{r}}(R)$. (For any $n, E_{n}(R)$ will denote the elementary subgroup of $S l_{n}(R)$ generated by the elementary generators $E_{i j}(\lambda), \lambda \in R, 1 \leq i \neq j \leq n$, which is the matrix with 1's on the diagonal, the $(i, j)$-th entry $\lambda$, and the rest of the entries to be zero. An element of $E_{n}(R)$ will be called an elementary matrix.)

The Suslin matrices have proved useful in several contexts. We mention some examples. (Only the first and third results mentioned below have been published so far.)

- A. Suslin showed that, for a field $k$,

$$
S K_{1}\left(\frac{k\left[x_{1}, \cdots, x_{n}, y_{1}, \cdots, y_{n}\right]}{\left(\sum_{i=1}^{n} x_{i} y_{i}-1\right)}\right) \simeq \mathbb{Z},
$$

with generator $\left[S_{n-1}\left(\left(x_{1}, \cdots, x_{n}\right),\left(y_{1}, \cdots, y_{n}\right)\right)\right]$.

- Let $\sum_{i=1}^{n} x_{i} y_{i}=1$. Let $P, P^{*}$ be the projective modules corresponding to the unimodular rows $\left(x_{1}, \cdots, x_{n}\right),\left(y_{1}, \cdots, y_{n}\right)$, respectively. Then $P^{*} \simeq \operatorname{Hom}_{R}(P, R)$, the dual of $P$. If $n$ is even, then $P \simeq P^{*}$. However, if $n>1$ is odd, then M.V. Nori, and R.G. Swan independently showed (using topological arguments) that $P, P^{*}$ need not be isomorphic. This can also be shown using the Suslin matrices, when $n>3$.

Received by the editors January 10, 2004 and, in revised form, July 19, 2004.

2000 Mathematics Subject Classification. Primary 13D15, 15A66, 19A15, 19E20, 55Q55.

This article is part of the first author's doctoral dissertation.

(C)2005 American Mathematical Society 
- Inspired by the proof of Serre's conjecture on the freeness of projective modules over a polynomial extension $k\left[x_{1}, \cdots, x_{n}\right]$ of a field $k$, and N. Mohan Kumar's theorems on set theoretic complete intersections, M. Boratýnski showed that any ideal $I$ in a polynomial ring $R$ over a field can be generated up to radical by $m=\mu\left(I / I^{2}\right)$ elements. Here $\mu$ denotes the minimal number of generators of the $R / I$-module $I / I^{2}$. Thus, one has $\sqrt{I}=\sqrt{\left(f_{1}, \cdots, f_{m}\right)}$, for some $f_{1}, \cdots, f_{m} \in R$.

- The orbit spaces $U m_{n}(R) / E_{n}(R)$ have a nice Witt group structure, if $R$ is a noetherian ring of Krull dimension $d$, in which, say, -1 is a square, and if $n \geq$ $\max \{3,(d+3) / 2\}$.

The key question raised by A. Suslin in [4] was whether these matrices had a role in defining a symbol on the orbits space $U m_{n}(R) / E_{n}(R)$, similar to the role of the Vaserstein symbol defined in $\underline{3}$, when $n=3$ and $\operatorname{dim} R=2$. This has surfaced partially in the last application mentioned above, due to the study initiated in this paper.

This paper arose out of 'a desire to play with the Suslin matrices'. Due to the inspiring [4, §5] there are several ways in which one can proceed to do this. We choose one such natural way in this article. With this aim, we introduce and study the subgroup $S U m_{r}(R)$ of $S l_{2^{r}}(R)$, which we christen the Special Unimodular Vector group, generated by the Suslin matrices $S_{r}(v, w)$, corresponding to the pairs $(v, w)$, with $v \cdot w^{T}=1$. Analogous to the elementary subgroup $E_{n}(R)$ we consider the Elementary Unimodular Vector subgroup $E U m_{r}(R)$, of $S U m_{r}(R)$, generated by the matrices $S_{r}(v, w)$ corresponding to the pair $(v, w)$, with $v \cdot w^{T}=1$, and with $v$ the first row of an elementary matrix. We develop the basic calculus for this group. As a consequence, we realize an important fundamental property of the Suslin matrices. (We exploit this property, and the equivalent key lemma here, to study the orbit space of unimodular vectors under elementary action, in a subsequent article.)

\section{Elementary orbit EQUALS COHN ORBIT}

Let $R$ be a commutative ring with identity 1 . Let $U m_{r+1}(R)$ denote the set of all unimodular vectors $v=\left(a_{0}, a_{1}, \cdots, a_{r}\right)$, i.e. $\sum a_{i} b_{i}=1$, for some $b_{i} \in R$, $0 \leq i \leq r$.

The vector $w=\left(b_{0}, b_{1}, \cdots, b_{r}\right)$ is said to be related to $v$ if the dot product $v \cdot w^{T}=1$. Note that vectors related to the same vector lie in the same elementary orbit, if their length is at least 3: If $v \cdot w_{1}^{T}=1=v \cdot w_{2}^{T}$, then $\varepsilon=I_{r+1}+v^{T}\left(w_{2}-w_{1}\right) \in$ $E_{r+1}(R)$ by [5. Corollary 2.7], and $w_{1} \varepsilon=w_{2}$.

Definition. Let $v=\left(a_{0}, a_{1}, \cdots, a_{r}\right)$ and $w=\left(b_{0}, b_{1}, \cdots, b_{r}\right)$ with $v \cdot w^{T}=1$. We say that the vector

$$
v^{*}=v C_{i j}(\lambda)=\left(a_{0}, \cdots, a_{i}+\lambda b_{j}, \cdots, a_{j}-\lambda b_{i}, \cdots, a_{r}\right),
$$

for $0 \leq i \neq j \leq r$, is a Cohn transform of $v$ w.r.t. the (related) vector $w$. One could write $C_{i j}(w, \lambda)$ if one wishes to specify $w$, but we generally refrain from doing so, as the related vector being considered is clear from the context. We shall say that a vector $v^{*}$ is in the Cohn orbit of $v$ if there is a related vector $w^{*}$ to $v^{*}$, and a sequence of pairs, starting with $\left(v_{0}, w_{0}\right)=(v, w)$ and ending with $\left(v_{r}, w_{r}\right)=\left(v^{*}, w^{*}\right)$, such that, for $i \geq 0$, the pairs $\left(v_{i+1}, w_{i+1}\right)$ have either $v_{i+1}$ as a Cohn transform of $v_{i}$ w.r.t. $w_{i}$, and $w_{i+1}=w_{i}$; or $w_{i+1}$ as a Cohn transform of $w_{i}$ w.r.t. $v_{i}$, and 
$v_{i+1}=v_{i}$ :

$$
(v, w)=\left(v_{0}, w_{0}\right) \rightarrow\left(v_{1}, w_{1}\right) \rightarrow \cdots \rightarrow\left(v_{r}, w_{r}\right)=\left(v^{*}, w^{*}\right) .
$$

It follows inductively, by the above observation, that $v^{*} \in v E_{r+1}(R)$, i.e. Cohn transforms lie in the same elementary orbit. Conversely, any elementary transformation of a vector can be obtained by means of a sequence of Cohn transforms w.r.t. some suitable related vectors. We show this explicitly below in a special case, when $r=2$. The general case can be easily reduced to the case when $r=2$, and we leave this for the reader to rediscover for himself.

Let $a a^{\prime}+b b^{\prime}+c c^{\prime}=1$. We show that $(a, b, c) \longrightarrow(a+\lambda b, b, c)$ can obtain a sequence of Cohn transforms. The idea comes from analysing the proof of 3 , Lemma 5.1]:

$$
\begin{array}{rll}
\left\{(a, b, c) ;\left(a^{\prime}, b^{\prime}, c^{\prime}\right)\right\} \stackrel{C_{02}(-\lambda)}{\longrightarrow} & \left\{\left(a-\lambda c^{\prime}, b, c+\lambda a^{\prime}\right) ;\left(a^{\prime}, b^{\prime}, c^{\prime}\right)\right\} \\
& \stackrel{C_{12}(-1)}{\longrightarrow} & \left\{\left(a-\lambda c^{\prime}, b, c+\lambda a^{\prime}\right) ;\left(a^{\prime}, b^{\prime}-\left(c+\lambda a^{\prime}\right), c^{\prime}+b\right)\right\} \\
& \stackrel{C_{02}(\lambda)}{\longrightarrow} & \left\{\left(\left(a-\lambda c^{\prime}\right)+\lambda\left(c^{\prime}+b\right), b,\left(c+\lambda a^{\prime}\right)-\lambda a^{\prime}\right) ;\right. \\
& \left.\left(a^{\prime}, b^{\prime}-\left(c+\lambda a^{\prime}\right), c^{\prime}+b\right)\right\} \\
= & \left\{(a+\lambda b, b, c) ;\left(a^{\prime}, b^{\prime}-c-\lambda a^{\prime}, c^{\prime}+b\right)\right\} \\
& \stackrel{C_{12}(1)}{\longrightarrow} & \left\{(a+\lambda b, b, c) ;\left(a^{\prime}, b^{\prime}-\lambda a^{\prime}, c^{\prime}\right)\right\} .
\end{array}
$$

Thus, one proves that

Lemma 2.1. The elementary orbit $v E_{r+1}(R)$ of $v \in U m_{r+1}(R)$ coincides with the Cohn orbit of $v$, for $r \geq 2$.

\section{The Unimodular VECTOR GROUPS}

We begin by describing the inductive process by which the Suslin matrix $S_{r}(v, w)$ of size $2^{r}$, and of determinant $v \cdot w^{T}$, is constructed from two vectors $v, w$ of size $r+1$. We recall this process.

Let $v=\left(a_{0}, v_{1}\right), w=\left(b_{0}, w_{1}\right)$. Set $S_{0}(v, w)=a_{0}$, and set

$$
S_{r}(v, w)=\left(\begin{array}{cc}
a_{0} I_{2^{r-1}} & S_{r-1}\left(v_{1}, w_{1}\right) \\
-S_{r-1}\left(w_{1}, v_{1}\right)^{T} & b_{0} I_{2^{r-1}}
\end{array}\right) .
$$

In [4, Lemma 5.1] it is noted that

(1) $S_{r}(v, w) S_{r}(w, v)^{T}=\left(v \cdot w^{T}\right) I_{2^{r}}=S_{r}(w, v)^{T} S_{r}(v, w)$, and

(2) det $S_{r}(v, w)=\left(v \cdot w^{T}\right)^{2^{r-1}}$, for $r \geq 1$.

A. Suslin then describes a sequence of forms $J_{r} \in M_{2^{r}}(R)$ by the recurrence formulae

$$
J_{r}= \begin{cases}1, & \text { for } r=0, \\ J_{r-1} \perp-J_{r-1}, & \text { for } r \text { even, } \\ J_{r-1} \top-J_{r-1}, & \text { for } r \text { odd. }\end{cases}
$$

(The English translation wrongly says $J_{r}=J_{r-1} \perp J_{r-1}$ when $r$ is even.)

(Here, for $\alpha \in M_{r, s}(R), \beta \in M_{p, q}(R), \alpha \perp \beta=\left(\begin{array}{cc}\alpha & 0 \\ 0 & \beta\end{array}\right)$, while $\alpha \top \beta=\left(\begin{array}{cc}0 & \alpha \\ \beta & 0\end{array}\right) \in$ $M_{r+p, s+q}(R)$.)

It is easy to see that $\operatorname{det} J_{r}=1$, for all $r$, and that $J_{r}^{T}=J_{r}^{-1}=(-1)^{\frac{r(r+1)}{2}} J_{r}$. Moreover, $J_{r}$ is antisymmetric if $r=4 k+1$ and $r=4 k+2$, whereas $J_{r}$ is symmetric 
for $r=4 k$ and $r=4 k+3$. In [4. Lemma 5.3], it is noted that the following formulae (called the Suslin identities) are valid:

$$
\begin{aligned}
\text { for } r=4 k:\left(S_{r}(v, w) J_{r}\right)^{T} & =S_{r}(v, w) J_{r} ; \\
\text { for } r=4 k+1: S_{r}(v, w) J_{r} S_{r}(v, w)^{T} & =\left(v \cdot w^{T}\right) J_{r} ; \\
\text { for } r=4 k+2:\left(S_{r}(v, w) J_{r}\right)^{T} & =-S_{r}(v, w) J_{r} ; \\
\text { for } r=4 k+3: S_{r}(v, w) J_{r} S_{r}(v, w)^{T} & =\left(v \cdot w^{T}\right) J_{r} .
\end{aligned}
$$

Definition. The Special Unimodular Vector group $S_{U} m_{r}(R)$ is the subgroup of $S l_{2^{r}}(R)$ generated by the Suslin matrices $S_{r}(v, w)$ w.r.t. the pair $(v, w)$, with $v \in U m_{r+1}(R)$, and for some $w$ related to $v$, i.e. $w \cdot v^{T}=1$.

The Elementary Unimodular Vector group $\operatorname{EUm}_{r}(R)$ is the subgroup of $\operatorname{SUm}_{r}(R)$ generated by the Suslin matrices $S_{r}(v, w)$, with $v \in e_{1} E_{r+1}(R)$, and with $w \cdot v^{T}=1$.

We shall denote by $\operatorname{EUm}_{r}(R)^{*}$ the subgroup of $E U m_{r}(R)$ generated by the elements $S_{r}\left(e_{1}+\lambda e_{i}, e_{1}\right), S_{r}\left(e_{1}, e_{1}+\lambda e_{i}\right)$, with $1<i \leq r+1, \lambda \in R$.

Let us first note a few preliminary observations.

Lemma 3.1. Let $R$ be a commutative ring and let $v, w, s, t \in R^{r+1}$. Let $v=$ $\left(a_{0}, a_{1}, \cdots, a_{r}\right), w=\left(b_{0}, b_{1}, \cdots, b_{r}\right)$. Then

$$
\begin{aligned}
S_{r}(v, w)+S_{r}(w, v)^{T} & =\left\{a_{0}+b_{0}\right\} I_{2^{r}}, \\
S_{r}(s, t) S_{r}(w, v)^{T}+S_{r}(v, w) S_{r}(t, s)^{T} & =\{\langle s, w\rangle+\langle v, t\rangle\} I_{2^{r}}, \\
S_{r}(w, v)^{T} S_{r}(s, t)+S_{r}(t, s)^{T} S_{r}(v, w) & =\{\langle s, w\rangle+\langle v, t\rangle\} I_{2^{r}} .
\end{aligned}
$$

Proof. One has an inner product on $R^{r+1} \times R^{r+1}$ defined by $\langle v, w\rangle=v \cdot w^{T}$. Also one has the usual bilinear consequence of the quadratic relation

$$
\langle v, w\rangle I_{2^{r}}=S_{r}(v, w) S_{r}(w, v)^{T}=S_{r}(w, v)^{T} S_{r}(v, w),
$$

viz.

$$
\begin{aligned}
S_{r}(v+s, w+t) S_{r}(w+t, v+s)^{T} & =\langle v+s, w+t\rangle I_{2^{r}} \\
& =\{\langle v, w\rangle+\langle v, t\rangle+\langle s, w\rangle+\langle s, t\rangle\} I_{2^{r}} .
\end{aligned}
$$

But

$$
\begin{aligned}
S_{r} & (v+s, w+t) S_{r}(w+t, v+s)^{T} \\
\quad & =\left\{S_{r}(v, w)+S_{r}(s, t)\right\}\left\{S_{r}(w, v)^{T}+S_{r}(t, s)^{T}\right\} \\
& =S_{r}(v, w) S_{r}(w, v)^{T}+S_{r}(v, w) S_{r}(t, s)^{T}+S_{r}(s, t) S_{r}(w, v)^{T}+S_{r}(s, t) S_{r}(t, s)^{T} \\
& =\langle v, w\rangle I_{2^{r}}+S_{r}(v, w) S_{r}(t, s)^{T}+S_{r}(s, t) S_{r}(w, v)^{T}+\langle s, t\rangle I_{2^{r}} .
\end{aligned}
$$

Equating the above two equations, we get

$$
S_{r}(s, t) S_{r}(w, v)^{T}+S_{r}(v, w) S_{r}(t, s)^{T}=\{\langle s, w\rangle+\langle v, t\rangle\} I_{2^{r}} .
$$

Similarly, one can prove the third relation. The first relation is obtained from the second by considering $s=t=e_{1}$.

Notation. For a matrix $\alpha \in M_{k}(R)$, we define $\alpha^{t o p}$ as the matrix whose entries are the same as those of $\alpha$ above the diagonal, and on the diagonal, and is zero below the diagonal. Similarly, we define $\alpha^{\text {bot }}$.

For simplicity we may write $\alpha^{t}$ for $\alpha^{t o p}, \alpha^{b}$ for $\alpha^{b o t}$, and $\alpha^{T}$ for $\alpha$ transpose. Moreover, we use $\alpha^{t b}$ for $\alpha^{t o p}$ or $\alpha^{b o t}$. Similarly, we write $E U m_{r}(R)^{t b}$ to be the 
subgroup of $E_{2^{r}}(R)$ generated by the elements $\alpha^{t b}$, for $\alpha$ a basic generator of $E U m_{r}(R)^{*}$.

We also write expressions like $x \alpha^{t b} x^{-1}=\beta^{b t}$ to mean that both $x \alpha^{t o p} x^{-1}=\beta^{b o t}$ and $x \alpha^{b o t} x^{-1}=\beta^{\text {top }}$ hold.

We now prove the key lemma.

Lemma 3.2. Let $v=\left(a_{0}, a_{1}, \cdots, a_{r}\right)=\left(a_{0}, v_{1}\right), w=\left(b_{0}, b_{1}, \cdots, b_{r}\right)=\left(b_{0}, w_{1}\right)$, with $v \cdot w^{T}=1$. Then, for $2 \leq i \leq r+1, r \geq 2$,

$$
\begin{aligned}
& S_{r}\left(e_{1}, e_{1}+\lambda e_{i}\right)^{t o p} S_{r}(v, w) S_{r}\left(e_{1}, e_{1}+\lambda e_{i}\right)^{b o t}=S_{r}\left(v E_{i 1}(-\lambda), w E_{1 i}(\lambda)\right), \\
& S_{r}\left(e_{1}+\lambda e_{i}, e_{1}\right)^{b o t} S_{r}(v, w) S_{r}\left(e_{1}+\lambda e_{i}, e_{1}\right)^{t o p}=S_{r}\left(v E_{1 i}(\lambda), w E_{i 1}(-\lambda)\right) .
\end{aligned}
$$

(The case when $r=1$ is similar.) Moreover, if $1 \leq i \leq r$, then

$$
\begin{aligned}
& S_{r}\left(e_{1}+\lambda e_{i+1}, e_{1}\right)^{t o p} S_{r}(v, w) S_{r}\left(e_{1}+\lambda e_{i+1}, e_{1}\right)^{b o t}=S_{r}\left(v C_{0 i}(-\lambda), w\right), \\
& S_{r}\left(e_{1}, e_{1}+\lambda e_{i+1}\right)^{b o t} S_{r}(v, w) S_{r}\left(e_{1}, e_{1}+\lambda e_{i+1}\right)^{t o p}=S_{r}\left(v, w C_{0 i}(-\lambda)\right) .
\end{aligned}
$$

Proof. As $S_{r}(v, w)^{-1}=S_{r}(w, v)^{T}$, one needs to verify the first and third of the above identities, in order to deduce the other two identities. Both these identities are derived similarly, by means of a direct computation. We derive them as follows:

$$
S_{r}\left(e_{1}, e_{1}+\lambda e_{i}\right)^{t o p} S_{r}(v, w) S_{r}\left(e_{1}, e_{1}+\lambda e_{i}\right)^{b o t}=\left(\begin{array}{ll}
\alpha_{11} & \alpha_{12} \\
\alpha_{21} & \alpha_{22}
\end{array}\right),
$$

where

$$
\begin{aligned}
\alpha_{11}= & a_{0} I_{2^{r-1}}-S_{r-1}\left(0, \lambda e_{i-1}\right) S_{r-1}\left(w_{1}, v_{1}\right)^{T}-S_{r-1}\left(v_{1}, w_{1}\right) S_{r-1}\left(\lambda e_{i-1}, 0\right)^{T} \\
& -b_{0} S_{r-1}\left(0, \lambda e_{i-1}\right) S_{r-1}\left(\lambda e_{i-1}, 0\right)^{T} \\
\alpha_{12}= & S_{r-1}\left(v_{1}, w_{1}\right)+b_{0} S_{r-1}\left(0, \lambda e_{i-1}\right) \\
\alpha_{21}= & -S_{r-1}\left(w_{1}, v_{1}\right)^{T}-b_{0} S_{r-1}\left(\lambda e_{i-1}, 0\right)^{T} \\
\alpha_{22}= & b_{0} I_{2^{r-1}} .
\end{aligned}
$$

Let $w^{*}=w E_{1 i}(\lambda)=\left(b_{0}, w_{1}^{*}\right)$. Hence, via Lemma 3.1, the above matrix equals

$$
\left(\begin{array}{cc}
\left(a_{0}-\lambda a_{i-1}\right) I_{2^{r-1}} & S_{r-1}\left(v_{1}, w_{1}^{*}\right) \\
-S_{r-1}\left(w_{1}^{*}, v_{1}\right)^{T} & b_{0} I_{2^{r-1}}
\end{array}\right)=S_{r}\left(v E_{i 1}(-\lambda), w E_{1 i}(\lambda)\right),
$$

as required. We now prove the third identity.

$$
S_{r}\left(e_{1}+\lambda e_{i+1}, e_{1}\right)^{t o p} S_{r}(v, w) S_{r}\left(e_{1}+\lambda e_{i+1}, e_{1}\right)^{b o t}=\left(\begin{array}{ll}
\alpha_{11} & \alpha_{12} \\
\alpha_{21} & \alpha_{22}
\end{array}\right),
$$

where

$$
\begin{aligned}
\alpha_{11}= & a_{0} I_{2^{r-1}}-S_{r-1}\left(\lambda e_{i}, 0\right) S_{r-1}\left(w_{1}, v_{1}\right)^{T}-S_{r-1}\left(v_{1}, w_{1}\right) S_{r-1}\left(\lambda e_{i}, 0\right) \\
& -S_{r-1}\left(\lambda e_{i}, 0\right) S_{r-1}\left(0, \lambda e_{i}\right)^{T} \\
\alpha_{12}= & S_{r-1}\left(v_{1}, w_{1}\right)+b_{0} S_{r-1}\left(\lambda e_{i}, 0\right) \\
\alpha_{21}= & -S_{r-1}\left(w_{1}, v_{1}\right)^{T}-b_{0} S_{r-1}\left(0, \lambda e_{i}\right)^{T} \\
\alpha_{22}= & b_{0} I_{2^{r-1}} .
\end{aligned}
$$

Let $v_{1}^{*}=\left(a_{1}, \cdots, a_{i}+\lambda b_{0}, \cdots, a_{r}\right)$. Then,

$$
\left(\begin{array}{cc}
\alpha_{11} & \alpha_{12} \\
\alpha_{21} & \alpha_{22}
\end{array}\right)=\left(\begin{array}{cc}
\left(a_{0}-\lambda b_{i}\right) I_{2^{r-1}} & S_{r-1}\left(v_{1}^{*}, w_{1}\right) \\
-S_{r-1}\left(w_{1}, v_{1}^{*}\right)^{T} & b_{0} I_{2^{r-1}}
\end{array}\right) .
$$

This equals $S_{r}\left(v C_{0 i}(-\lambda), w\right)$, as required. 
Remark. Note that we shall always regard $v=\left(a_{0}, a_{1}, \cdots, a_{r}\right) \in M_{1 r+1}(R)$, as $\sum_{i=0}^{r} a_{i} e_{i+1}$. Thus, for instance, $e_{1}+\lambda e_{2}=(1, \lambda, \cdots, 0)$, etc. It will be convenient to use the following notation later: For $1 \leq i \leq r+1, \lambda \in R, \lambda$ unit if $i=1$,

$$
\begin{aligned}
& E\left(e_{1}\right)(\lambda)=\lambda I_{2^{r-1}} \perp \lambda^{-1} I_{2^{r-1}}, \\
& E\left(e_{i}\right)(\lambda)=S_{r}\left(e_{1}+\lambda e_{i}, e_{1}\right) ; i>1, \\
& E\left(e_{i}^{*}\right)(\lambda)=S_{r}\left(e_{1}, e_{1}+\lambda e_{i}\right) ; i>1 .
\end{aligned}
$$

(If we wish to stress the size we could write $E_{r}\left(e_{i}\right)(\lambda), E_{r}\left(e_{i}^{*}\right)(\lambda)$.)

Note also that $E\left(e_{i}\right)(\lambda)^{T}=E\left(e_{i}^{*}\right)(-\lambda)$ and $E\left(e_{i}^{*}\right)(\lambda)^{T}=E\left(e_{i}\right)(-\lambda)$ hold due to the "anti-symmetry" of the Suslin matrices.

The key lemma leads one to the fundamental property of Suslin matrices, via the methods of commutative algebra.

Corollary 3.3. Let $R$ be a commutative ring in which 2 is invertible. Let $\alpha=$ $S_{1} S_{2} \cdots S_{n}$ and $\alpha^{*}=S_{n} S_{n-1} \cdots S_{1}$, where each $S_{i}$ is a Suslin matrix $S_{r}\left(v_{i}, w_{i}\right)$, with $\left\langle v_{i}, w_{i}\right\rangle=1$. For any $\beta=S_{r}(v, w), \alpha \beta \alpha^{*}=S_{r}\left(v^{\prime}, w^{\prime}\right) \in S U m_{r}(R)$.

Proof. Let $\wp$ be a prime ideal of $R$. Let $S_{i}=S_{r}\left(v_{i}, w_{i}\right)$, for $v_{i}, w_{i} \in R^{r+1},\left\langle v_{i}, w_{i}\right\rangle=$ $v_{i} \cdot w_{i}^{T}=1$. Note that $U m_{r+1}\left(R_{\wp}\right)=e_{1} E_{r+1}\left(R_{\wp}\right)$. Using the observation at the beginning of Section 2, one can conclude that there is a $\varepsilon \in E_{r+1}\left(R_{\wp}\right)$ with $e_{1} \varepsilon=v$, and with $e_{1} \varepsilon^{T^{-1}}=w$. Each $\varepsilon_{i}$ can be written as a product of elementary generators of type $E_{1 j}(\lambda), E_{j 1}(\mu)$, for $2 \leq j \leq r+1, \lambda, \mu \in R$. Hence, by Lemma 3.2,

$$
S_{r}\left(v_{i}, w_{i}\right)=S_{r}\left(e_{1} \varepsilon_{i}, e_{1} \varepsilon_{i}^{T^{-1}}\right)=\sigma_{1 i}^{t b} \cdots \sigma_{l_{i} i}^{t b} I_{2^{r}} \sigma_{l_{i} i}^{b t} \cdots \sigma_{1 i}^{b t},
$$

where $\sigma_{p q}^{t b}$ is a generator of type $E(c)(\lambda)^{t b}$, for $c=e_{t}$ or $e_{t}^{*}, \lambda \in R$.

Observe that over $R_{\wp}$,

$$
\begin{aligned}
S_{r}\left(v_{i}, w_{i}\right) S_{r}(x, y) S_{r}\left(v_{i}, w_{i}\right) & =\sigma_{1 i}^{t b} \cdots \sigma_{l_{i} i}^{t b} \sigma_{l_{i} i}^{b t} \cdots \sigma_{1 i}^{b t} S_{r}(x, y) \sigma_{1 i}^{t b} \cdots \sigma_{l_{i} i}^{t b} \sigma_{l_{i} i}^{b t} \cdots \sigma_{1 i}^{b t} \\
& =S_{r}\left(x^{\prime}, y^{\prime}\right),
\end{aligned}
$$

by Lemma 3.2, with $\left\langle x^{\prime}, y^{\prime}\right\rangle=1$. From this it follows that $\alpha \beta \alpha^{*}$ "locally looks like" an $S_{r}\left(v^{\prime}, w^{\prime}\right)$ with $\left\langle v^{\prime}, w^{\prime}\right\rangle=1$.

Hence, $\alpha \beta \alpha^{*}$ has a certain configuration amongst its entries. The reader should be able to convince him/herself that indeed $\alpha \beta \alpha^{*}=S_{r}\left(v^{\prime}, w^{\prime}\right)$, for some $v^{\prime}, w^{\prime}$ with $\left\langle v^{\prime}, w^{\prime}\right\rangle=1$.

Remark 3.4. A direct proof of the fundamental property can be obtained via Lemma 3.1. We have included this in 2, where the property is studied in greater detail.

The usual elementary generators $E_{i j}(\lambda), i \neq j, \lambda \in R$, of the elementary subgroup of the linear group, and the elementary symplectic generators $S_{i j}(\lambda), i \neq$ $j, \lambda \in R$, have certain "splitting properties". These are reflected in the basic elementary generators of $E U m_{r}(R)^{*}$, viz.

Lemma 3.5. Let $R$ be a commutative ring. For $2 \leq i \leq r+1, \lambda, \mu \in R$,

$$
\begin{aligned}
E\left(e_{i}\right)(\lambda+\mu) & =E\left(e_{i}\right)(\lambda) E\left(e_{i}\right)(\mu), \\
E\left(e_{i}^{*}\right)(\lambda+\mu) & =E\left(e_{i}^{*}\right)(\lambda) E\left(e_{i}^{*}\right)(\mu), \\
E\left(e_{i}\right)(\lambda) & =E\left(e_{i}\right)(\lambda)^{t o p} E\left(e_{i}\right)(\lambda)^{b o t}=E\left(e_{i}\right)(\lambda)^{b o t} E\left(e_{i}\right)(\lambda)^{t o p}, \\
E\left(e_{i}^{*}\right)(\lambda) & =E\left(e_{i}^{*}\right)(\lambda)^{t o p} E\left(e_{i}^{*}\right)(\lambda)^{b o t}=E\left(e_{i}^{*}\right)(\lambda)^{b o t} E\left(e_{i}^{*}\right)(\lambda)^{t o p} .
\end{aligned}
$$


Proof. These will follow by a direct computation using Lemma 3.1.

The next lemma considers some typical examples.

Lemma 3.6. Let $2 \leq i \neq j \leq r+1$, and let $\lambda=-2 x y$. Then:

(i) If $\alpha=\left[E\left(e_{i}\right)(x), E\left(e_{j}\right)(y)\right]$ and $\alpha^{*}=\left[E\left(e_{j}\right)(-y), E\left(e_{i}\right)(-x)\right]$, then $\alpha^{*}=$ $\alpha^{-1}$, and $S_{r}\left(v C_{i-1 j-1}(\lambda), w\right)=\alpha S_{r}(v, w) \alpha^{-1}$.

(ii) If $\beta=\left[E\left(e_{i}^{*}\right)(x), E\left(e_{j}^{*}\right)(y)\right]$ and $\beta^{*}=\left[E\left(e_{j}^{*}\right)(-y), E\left(e_{i}^{*}\right)(-x)\right]$, then $\beta^{*}=$ $\beta^{-1}$, and $S_{r}\left(v, w C_{i-1 j-1}(\lambda)\right)=\beta S_{r}(v, w) \beta^{-1}$.

(iii) If $\gamma=\left[E\left(e_{j}\right)(x), E\left(e_{i}^{*}\right)(y)\right]$ and $\gamma^{*}=\left[E\left(e_{i}^{*}\right)(-y), E\left(e_{j}\right)(-x)\right]$, then $\gamma^{*}=$ $\gamma^{-1}$, and $S_{r}\left(v E_{i j}(\lambda), w E_{j i}(-\lambda)\right)=\gamma S_{r}(v, w) \gamma^{-1}$.

Proof. The reader may consider the following approaches to prove the above lemma: Directly verify via Lemma 3.2 , or do direct computations with the matrices and use observations as in Lemma 3.1.

\section{Commutator laws in $E U m_{r}(R)^{*}$}

We next state and prove the "commutator laws" in $E U m_{r}(R)^{*}$. The basic instinct of these formulae is to convey the information that the commutator of an elementary unimodular generator in $\mathbb{Z}[\lambda]$, and an elementary unimodular generator in $\mathbb{Z}[\mu]$, will be an elementary unimodular generator in $\mathbb{Z}[\lambda \mu]$.

Lemma 4.1. For $1<i<j \leq r+1, \lambda, \mu \in R, c_{i}=e_{i}$ or $e_{i}^{*}, d_{j}=e_{j}$ or $e_{j}^{*}$, we have, for $r \geq 2$,

$$
\begin{aligned}
& {\left[E_{r}\left(c_{i}\right)(\lambda), E_{r}\left(d_{j}\right)(\mu)\right]} \\
& \quad=\left[E_{r-1}\left(c_{i-1}\right)(\lambda), E_{r-1}\left(d_{j-1}\right)(\mu)\right] \perp\left[E_{r-1}\left(c_{i-1}\right)(\lambda), E_{r-1}\left(d_{j-1}\right)(\mu)\right] \\
& \quad=\alpha \perp \cdots \perp \alpha,
\end{aligned}
$$

where

$$
\alpha= \begin{cases}\left\{E_{r-i+1}\left(d_{j-i+1}\right)(2 \lambda \mu)^{t o p} \perp E_{r-i+1}\left(d_{j-i+1}\right)(-2 \lambda \mu)^{b o t}\right\} & \text { if } c_{i}=e_{i}, \\ \left\{E_{r-i+1}\left(d_{j-i+1}\right)(2 \lambda \mu)^{b o t} \perp E_{r-i+1}\left(d_{j-i+1}\right)(-2 \lambda \mu)^{t o p}\right\} & \text { if } c_{i}=e_{i}^{*} .\end{cases}
$$

(The $\alpha$ is taken $2^{i-2}$ times above.)

Proof. We only prove here the first result when $c_{i}=e_{i}$, and $d_{j}=e_{j}$; the rest are proved similarly. We do it by induction on $i$. Note that

$$
\begin{aligned}
& {\left[E_{r}\left(e_{i}\right)(\lambda), E_{r}\left(e_{j}\right)(\mu)\right]} \\
& \left.\quad=\left[\begin{array}{cc}
I & S_{r-1}\left(\lambda e_{i-1}, 0\right) \\
-S_{r-1}\left(0, \lambda e_{i-1}\right)^{T} & I
\end{array}\right),\left(\begin{array}{cc}
I & S_{r-1}\left(\mu e_{j-1}, 0\right) \\
-S_{r-1}\left(0, \mu e_{j-1}\right)^{T} & I
\end{array}\right)\right] \\
& \quad=\left(\begin{array}{cc}
I-S_{r-1}\left(\lambda e_{i-1}, 0\right) S_{r-1}\left(0, \mu e_{j-1}\right)^{T} & S_{r-1}\left(\lambda e_{i-1}+\mu e_{j-1}, 0\right) \\
-S_{r-1}\left(0, \lambda e_{i-1}+\mu e_{j-1}\right)^{T} & I-S_{r-1}\left(0, \lambda e_{i-1}\right)^{T} S_{r-1}\left(\mu e_{j-1}, 0\right)
\end{array}\right) \\
& \quad \times\left(\begin{array}{cc}
I-S_{r-1}\left(\lambda e_{i-1}, 0\right) S_{r-1}\left(0, \mu e_{j-1}\right)^{T} & -S_{r-1}\left(\lambda e_{i-1}+\mu e_{j-1}, 0\right) \\
S_{r-1}\left(0, \lambda e_{i-1}+\mu e_{j-1}\right)^{T} & I-S_{r-1}\left(0, \lambda e_{i-1}\right)^{T} S_{r-1}\left(\mu e_{j-1}, 0\right)
\end{array}\right) .
\end{aligned}
$$

Via Lemma 3.1,

(i) $S_{r-1}\left(\lambda e_{i-1}, 0\right) S_{r-1}\left(0, \mu e_{j-1}\right)^{T} S_{r-1}\left(\lambda e_{i-1}, 0\right) S_{r-1}\left(0, \mu e_{j-1}\right)^{T}$

$$
\begin{aligned}
& =-S_{r-1}\left(\mu e_{j-1}, 0\right) S_{r-1}\left(0, \lambda e_{i-1}\right)^{T} S_{r-1}\left(\lambda e_{i-1}, 0\right) S_{r-1}\left(0, \mu e_{j-1}\right)^{T} \\
& =0 .
\end{aligned}
$$


(ii) $S_{r-1}\left(\lambda e_{i-1}, 0\right) S_{r-1}\left(0, \mu e_{j-1}\right)^{T} S_{r-1}\left(\lambda e_{i-1}+\mu e_{j-1}, 0\right)$

$$
\begin{aligned}
& =S_{r-1}\left(\lambda e_{i-1}, 0\right) S_{r-1}\left(0, \mu e_{j-1}\right)^{T}\left[S_{r-1}\left(\lambda e_{i-1}, 0\right)+S_{r-1}\left(\mu e_{j-1}, 0\right)\right] \\
& =S_{r-1}\left(\lambda e_{i-1}, 0\right) S_{r-1}\left(0, \mu e_{j-1}\right)^{T} S_{r-1}\left(\lambda e_{i-1}, 0\right) \\
& =-S_{r-1}\left(\mu e_{j-1}, 0\right) S_{r-1}\left(0, \lambda e_{i-1}\right)^{T} S_{r-1}\left(\lambda e_{i-1}, 0\right)=0 .
\end{aligned}
$$

Hence,

$$
\begin{aligned}
& {\left[E_{r}\left(e_{i}\right)(\lambda), E_{r}\left(e_{j}\right)(\mu)\right]} \\
& \quad=\left(\begin{array}{cc}
I-2 S_{r-1}\left(\lambda e_{i-1}, 0\right) S_{r-1}\left(0, \mu e_{j-1}\right)^{T} & 0 \\
0 & I-2 S_{r-1}\left(0, \lambda e_{i-1}\right)^{T} S_{r-1}\left(\mu e_{j-1}, 0\right)
\end{array}\right) .
\end{aligned}
$$

When $i=2$,

$$
\begin{aligned}
I-2 & S_{r-1}\left(\lambda e_{1}, 0\right) S_{r-1}\left(0, \mu e_{j-1}\right)^{T} \\
& =I-2\left(\begin{array}{cc}
\lambda I_{2^{r-2}} & 0 \\
0 & 0
\end{array}\right)\left(\begin{array}{cc}
0 & -S_{r-2}\left(\mu e_{j-2}, 0\right) \\
S_{r-2}\left(0, \mu e_{j-2}\right)^{T} & 0
\end{array}\right) \\
& =\left(\begin{array}{cc}
I_{2^{r-2}} & S_{r-2}\left(2 \lambda \mu e_{j-2}, 0\right) \\
0 & I_{2^{r-2}}
\end{array}\right)=E_{r-1}\left(e_{j-1}\right)(2 \lambda \mu)^{t o p} .
\end{aligned}
$$

Similarly, by direct computation, we can show that

$$
I-2 S_{r-1}\left(0, \lambda e_{i-1}\right)^{T} S_{r-1}\left(\mu e_{j-1}, 0\right)=E_{r-1}\left(e_{j-1}\right)(-2 \lambda \mu)^{b o t} .
$$

Hence $\left[E_{r}\left(e_{2}\right)(\lambda), E_{r}\left(e_{j}\right)(\mu)\right]=E_{r-1}\left(e_{j-1}\right)(2 \lambda \mu)^{t o p} \perp E_{r-1}\left(e_{j-1}\right)(-2 \lambda \mu)^{b o t}$.

Now consider the case when $i>2$. Via Lemma 3.1, we can write

$$
\begin{aligned}
& {\left[E_{r}\left(e_{i}\right)(\lambda), E_{r}\left(e_{j}\right)(\mu)\right]} \\
& \quad=\left(\begin{array}{cc}
I-2 S_{r-1}\left(\lambda e_{i-1}, 0\right) S_{r-1}\left(0, \mu e_{j-1}\right)^{T} & 0 \\
0 & I-2 S_{r-1}\left(\lambda e_{i-1}, 0\right) S_{r-1}\left(0, \mu e_{j-1}\right)^{T}
\end{array}\right) .
\end{aligned}
$$

Note that

$$
\begin{aligned}
& I-2 S_{r-1}\left(\lambda e_{i-1}, 0\right) S_{r-1}\left(0, \mu e_{j-1}\right)^{T} \\
& =I-2\left(\begin{array}{cc}
0 & S_{r-2}\left(\lambda e_{i-2}, 0\right) \\
-S_{r-2}\left(0, \lambda e_{i-2}\right)^{T} & 0
\end{array}\right)\left(\begin{array}{cc}
0 & -S_{r-2}\left(\mu e_{j-2}, 0\right) \\
S_{r-2}\left(0, \mu e_{j-2}\right)^{T} & 0
\end{array}\right) \\
& =\left(\begin{array}{cc}
I-2 S_{r-2}\left(\lambda e_{i-2}, 0\right) S_{r-2}\left(0, \mu e_{j-2}\right)^{T} & 0 \\
0 & I-2 S_{r-2}\left(0, \lambda e_{i-2}\right)^{T} S_{r-2}\left(\mu e_{j-2}, 0\right)
\end{array}\right) \\
& =\left[E_{r-1}\left(e_{i-1}\right)(\lambda), E_{r-1}\left(e_{j-1}\right)(\mu)\right] .
\end{aligned}
$$

Hence

$$
\begin{aligned}
& {\left[E_{r}\left(e_{i}\right)(\lambda), E_{r}\left(e_{j}\right)(\mu)\right]} \\
& \quad=\left[E_{r-1}\left(e_{i-1}\right)(\lambda), E_{r-1}\left(e_{j-1}\right)(\mu)\right] \perp\left[E_{r-1}\left(e_{i-1}\right)(\lambda), E_{r-1}\left(e_{j-1}\right)(\mu)\right] .
\end{aligned}
$$

The rest follows by a simple induction argument on $i$.

Corollary 4.2. For $2 \leq i \neq j \leq r+1$,

$$
\begin{aligned}
{\left[E\left(e_{i}\right)(\lambda), E\left(e_{j}^{*}\right)(\mu)\right] } & =\left[E\left(e_{i}\right)(x), E\left(e_{j}^{*}\right)(y)\right], \text { if } x y=\lambda \mu, \\
{\left[E\left(e_{i}\right)(\lambda), E\left(e_{j}\right)(\mu)\right] } & =\left[E\left(e_{i}\right)(x), E\left(e_{j}\right)(y)\right], \text { if } x y=\lambda \mu, \\
{\left[E\left(e_{i}^{*}\right)(\lambda), E\left(e_{j}^{*}\right)(\mu)\right] } & =\left[E\left(e_{i}^{*}\right)(x), E\left(e_{j}^{*}\right)(y)\right], \text { if } x y=\lambda \mu .
\end{aligned}
$$

Proof. This is obvious from the previous result. 
The next lemma plays the role in $E U m_{r}(R)^{*}$ of the role played by the commutator law $E_{i j}(\lambda \mu)=\left[E_{i k}(\lambda), E_{k j}(\mu)\right]$, for $\lambda, \mu \in R, 1 \leq i \neq j \neq k \leq r+1$, in calculations done in $E_{r+1}(R)$.

Lemma 4.3 (Biduality). For $2 \leq j \neq k \leq r+1$,

$$
\begin{aligned}
& E_{r}\left(e_{k}^{*}\right)(2 \lambda \mu \nu)\left[E\left(e_{j}\right)(\lambda), E\left(e_{k}^{*}\right)(\lambda \mu \nu)\right]=[x,[y, z]], \\
& E_{r}\left(e_{k}\right)(2 \lambda \mu \nu)\left[E\left(e_{j}^{*}\right)(\lambda), E\left(e_{k}\right)(\lambda \mu \nu)\right]=\left[x^{*},\left[y^{*}, z^{*}\right]\right],
\end{aligned}
$$

where $x=E_{r}\left(e_{j}\right)(\lambda), y=E_{r}\left(e_{k}^{*}\right)(\mu), z=E_{r}\left(e_{j}^{*}\right)(\nu), x^{*}=E_{r}\left(e_{j}^{*}\right)(\lambda), y^{*}=$ $E_{r}\left(e_{k}\right)(\mu), z^{*}=E_{r}\left(e_{j}\right)(\nu)$.

Proof. We establish the first equation, and the second can be done similarly.

First we consider the case when $j=2$. By Lemma 4.1, $[x,[y, z]]=[\alpha, \beta]$, where $\alpha=x$ and $\beta=E\left(e_{k-1}^{*}\right)(-2 \mu \nu)^{b o t} \perp E\left(e_{k-1}^{*}\right)(2 \mu \nu)^{t o p}$.

Now by direct computation, we get $[\alpha, \beta]=\left(\begin{array}{ll}A_{11} & A_{12} \\ A_{21} & A_{22}\end{array}\right)$, where

$$
\begin{aligned}
A_{11} & =I_{2^{r-1}}+S_{r-1}\left(\lambda e_{1}, 0\right) E\left(e_{k-1}^{*}\right)(2 \mu \nu)^{t o p} S_{r-1}\left(0, \lambda e_{1}\right)^{T} E\left(e_{k-1}^{*}\right)(-2 \mu \nu)^{b o t} \\
& =\left(\begin{array}{cc}
I_{2^{r-2}} & S_{r-2}\left(0,2 \lambda^{2} \mu \nu e_{k-2}\right) \\
0 & I_{2^{r-2}}
\end{array}\right)=E\left(e_{k-1}^{*}\right)\left(2 \lambda^{2} \mu \nu\right)^{t o p}, \\
A_{12} & =-E\left(e_{k-1}^{*}\right)(-2 \mu \nu)^{b o t} S_{r-1}\left(\lambda e_{1}, 0\right) E\left(e_{k-1}^{*}\right)(2 \mu \nu)^{t o p}+S_{r-1}\left(\lambda e_{1}, 0\right) \\
& =\left(\begin{array}{cc}
0 & S_{r-2}\left(0,2 \lambda \mu \nu e_{k-2}\right) \\
-S_{r-2}\left(2 \lambda \mu \nu e_{k-2}, 0\right)^{T} & 0
\end{array}\right)=S_{r-1}\left(0,2 \lambda \mu \nu e_{k-1}\right) \\
& =S_{r-1}\left(0,2 \lambda \mu \nu e_{k-1}\right) E\left(e_{k-1}^{*}\right)\left(-2 \lambda^{2} \mu \nu\right)^{b o t}, \\
A_{21} & =E\left(e_{k-1}^{*}\right)(2 \mu \nu)^{t o p} S_{r-1}\left(0, \lambda e_{1}\right)^{T} E\left(e_{k-1}^{*}\right)(-2 \mu \nu)^{b o t}-S_{r-1}\left(0, \lambda e_{1}\right)^{T} \\
& =\left(\begin{array}{cc}
0 & S_{r-2}\left(0,2 \lambda \mu \nu e_{k-2}\right) \\
-S_{r-2}\left(2 \lambda \mu \nu e_{k-2}, 0\right)^{T} & 0
\end{array}\right)=S_{r-1}\left(0,2 \lambda \mu \nu e_{k-1}\right) \\
& =-S_{r-1}\left(2 \lambda \mu \nu e_{k-1}, 0\right)^{T}=-S_{r-1}\left(2 \lambda \mu \nu e_{k-1}, 0\right)^{T} E\left(e_{k-1}^{*}\right)\left(2 \lambda^{2} \mu \nu\right)^{t o p}, \\
A_{22} & =I_{2^{r-1}}+S_{r-1}\left(0, \lambda e_{1}\right)^{T} E\left(e_{k-1}^{*}\right)(-2 \mu \nu)^{b o t} S_{r-1}\left(\lambda e_{1}, 0\right) E\left(e_{k-1}^{*}\right)(2 \mu \nu)^{t o p} \\
& =\left(\begin{array}{cc}
I_{2^{r-2}} & 0 \\
S_{r-2}\left(2 \lambda^{2} \mu \nu e_{k-2}, 0\right)^{T} & I_{2^{r-2}}
\end{array}\right)=E\left(e_{k-1}^{*}\right)\left(-2 \lambda^{2} \mu \nu\right)^{b o t} .
\end{aligned}
$$

Thus,

$[x,[y, z]]$

$$
\begin{aligned}
& =\left(\begin{array}{cc}
E\left(e_{k-1}^{*}\right)\left(2 \lambda^{2} \mu \nu\right)^{t o p} & S_{r-1}\left(0,2 \lambda \mu \nu e_{k-1}\right) E\left(e_{k-1}^{*}\right)\left(-2 \lambda^{2} \mu \nu\right)^{b o t} \\
-S_{r-1}\left(2 \lambda \mu \nu e_{k-1}, 0\right)^{T} E\left(e_{k-1}^{*}\right)\left(2 \lambda^{2} \mu \nu\right)^{t o p} & E\left(e_{k-1}^{*}\right)\left(-2 \lambda^{2} \mu \nu\right)^{b o t}
\end{array}\right) \\
& =E\left(e_{k}^{*}\right)(2 \lambda \mu \nu)\left[E\left(e_{2}\right)(\lambda), E\left(e_{k}^{*}\right)(\lambda \mu \nu)\right],
\end{aligned}
$$

as required. The last equalities in $A_{12}$, and $A_{21}$ above follow by a direct computation to show that

$$
\begin{aligned}
S_{r-1}\left(0,2 \lambda \mu \nu e_{k-1}\right) E\left(e_{k-1}^{*}\right)\left(-2 \lambda^{2} \mu \nu\right)^{b o t} & =S_{r-1}\left(0,2 \lambda \mu \nu e_{k-1}\right) \\
-S_{r-1}\left(2 \lambda \mu \nu e_{k-1}, 0\right)^{T} E\left(e_{k-1}^{*}\right)\left(2 \lambda^{2} \mu \nu\right)^{t o p} & =S_{r-1}\left(0,2 \lambda \mu \nu e_{k-1}\right) \\
& =-S_{r-1}\left(2 \lambda \mu \nu e_{k-1}, 0\right)^{T},
\end{aligned}
$$

as $k \neq 2$. The case when $k=2$ can be done similarly as above.

Now we consider the general case when $2<j \neq k \leq r+1$. 
By Lemma 4.1, $[x,[y, z]]=[\alpha, \beta]$, where $\alpha=x, \beta=A \perp A$, for $A=I-$ $2 S_{r-1}\left(0, \mu e_{k-1}\right) S_{r-1}\left(\nu e_{j-1}, 0\right)^{T}$. Moreover, $[\alpha, \beta]=\gamma \delta$, where

$$
\gamma=\left(\begin{array}{ll}
A & B \\
C & A
\end{array}\right), \delta=\left(\begin{array}{ll}
A^{\prime} & B^{\prime} \\
C^{\prime} & A^{\prime}
\end{array}\right),
$$

where $B=S_{r-1}\left(\lambda e_{j-1}, 0\right) A, C=-S_{r-1}\left(0, \lambda e_{j-1}\right)^{T} A, B^{\prime}=-S_{r-1}\left(\lambda e_{j-1}, 0\right) A^{\prime}$, $C^{\prime}=S_{r-1}\left(0, \lambda e_{j-1}\right)^{T} A^{\prime}, A^{\prime}=I+2 S_{r-1}\left(0, \mu e_{k-1}\right) S_{r-1}\left(\nu e_{j-1}, 0\right)^{T}$.

Note via Lemma 3.1 that

$$
\begin{aligned}
& S_{r-1}\left(0, \mu e_{k-1}\right) S_{r-1}\left(\nu e_{j-1}, 0\right)^{T} S_{r-1}\left(\lambda e_{j-1}, 0\right) \\
& \quad=-S_{r-1}\left(0, \mu e_{k-1}\right)\left[S_{r-1}\left(0, \lambda e_{j-1}\right)^{T} S_{r-1}\left(0, \nu e_{j-1}\right)-\lambda \nu I\right] \\
& \quad=S_{r-1}\left(\lambda e_{j-1}, 0\right) S_{r-1}\left(\mu e_{k-1}, 0\right)^{T} S_{r-1}\left(0, \nu e_{j-1}\right)+\lambda \nu S_{r-1}\left(0, \mu e_{k-1}\right) \\
& \quad=S_{r-1}\left(\lambda e_{j-1}, 0\right) S_{r-1}\left(0, \mu e_{k-1}\right) S_{r-1}\left(\nu e_{j-1}, 0\right)^{T}+\lambda \nu S_{r-1}\left(0, \mu e_{k-1}\right) .
\end{aligned}
$$

Therefore,

$$
\begin{aligned}
& S_{r-1}\left(\lambda e_{j-1}, 0\right)\left\{I-2 S_{r-1}\left(0, \mu e_{k-1}\right) S_{r-1}\left(\nu e_{j-1}, 0\right)^{T}\right\} \\
& \quad=\left\{I-2 S_{r-1}\left(0, \mu e_{k-1}\right) S_{r-1}\left(\nu e_{j-1}, 0\right)^{T}\right\} S_{r-1}\left(\lambda e_{j-1}, 0\right)-2 \lambda \nu S_{r-1}\left(\mu e_{k-1}, 0\right)^{T} .
\end{aligned}
$$

Hence for $\alpha=\left\{I-2 S_{r-1}\left(0, \mu e_{k-1}\right) S_{r-1}\left(\nu e_{j-1}, 0\right)^{T}\right\}$,

$$
S_{r-1}\left(\lambda e_{j-1}, 0\right) \alpha=\alpha S_{r-1}\left(\lambda e_{j-1}, 0\right)+2 \lambda \nu S_{r-1}\left(0, \mu e_{k-1}\right) .
$$

Therefore, as $S_{r-1}\left(\lambda e_{j-1}, 0\right)=-S_{r-1}\left(0, \lambda e_{j-1}\right)^{T}$,

$$
-S_{r-1}\left(0, \lambda e_{j-1}\right)^{T} \alpha=-\alpha S_{r-1}\left(0, \lambda e_{j-1}\right)^{T}+2 \lambda \nu S_{r-1}\left(0, \mu e_{k-1}\right) .
$$

Also for $\beta=\left\{I+2 S_{r-1}\left(0, \mu e_{k-1}\right) S_{r-1}\left(\nu e_{j-1}, 0\right)^{T}\right\}$, one can show that

$$
\begin{aligned}
-S_{r-1}\left(\lambda e_{j-1}, 0\right) \beta & =-\beta S_{r-1}\left(\lambda e_{j-1}, 0\right)+2 \lambda \nu S_{r-1}\left(0, \mu e_{k-1}\right), \\
S_{r-1}\left(0, \lambda e_{j-1}\right)^{T} \beta & =\beta S_{r-1}\left(0, \lambda e_{j-1}\right)^{T}+2 \lambda \nu S_{r-1}\left(0, \mu e_{k-1}\right) .
\end{aligned}
$$

Thus, using the above identities, for $\gamma=2 S_{r-1}\left(\lambda \mu \nu e_{k-1}, 0\right)^{T} S_{r-1}\left(\lambda e_{j-1}, 0\right)$ one can show that

$$
\begin{aligned}
{[x,[y, z]] } & =\left(\begin{array}{cc}
I+\gamma & S_{r-1}\left(0,2 \lambda \mu \nu e_{k-1}\right) \\
-S_{r-1}\left(2 \lambda \mu \nu e_{k-1}, 0\right)^{T} & I+\gamma
\end{array}\right) \\
& =E\left(e_{k}^{*}\right)(2 \lambda \mu \nu)\left[E\left(e_{j}\right)(\lambda), E\left(e_{k}^{*}\right)(\lambda \mu \nu)\right],
\end{aligned}
$$

as was claimed.

\section{Structure theorem for $E U m_{r}(R)$}

The elementary subgroup $E_{r}(R)$ of $G L_{\gamma}(R)$ is generated by the basic generators $E_{i j}(\lambda), 1 \leq i \neq j \leq r, \lambda \in R$. In view of the commutator laws, the generators $E_{1 i}(\lambda), E_{i 1}(\lambda), 2 \leq i \leq r, \lambda \in R$, suffices to generate $E_{r}(R)$.

Therefore, it seems natural to expect that the elementary unimodular generators $E\left(e_{i}\right)(\lambda), 2 \leq i \leq r+1, \lambda \in R$, should suffice to generate $\operatorname{EUm}_{r}(R)$. However, this is not the case, as a simple example will show: Note that elementary generators of type $E_{2}\left(e_{p}\right)(\mu), E_{2}\left(e_{q}\right)(\nu)$, are (elementary) symplectic. However, $S_{2}\left(e_{2}+e_{1}, e_{2}\right)$ is not symplectic, and so cannot be written as a product of these generators.

We examine below which additional elementary unimodular generators need to be added to the above set of generators, so as to suffice. The next two observations obviate the necessity of some generators. 
Lemma 5.1. Let $R$ be a commutative ring. For $1<i \neq j \leq r+1, \lambda \in R$, $S_{r}\left(e_{j}+\lambda e_{i}, e_{j}\right)$ can be written as a product of generators $E_{r}(c)(\lambda)$, for $c \in\left\{e_{i}, e_{i}^{*}\right.$, $\left.e_{j}, e_{j}^{*}\right\}$.

Proof. By Lemma 3.2,

$$
\begin{aligned}
S_{r}\left(e_{j}, e_{j}\right) & =E\left(e_{j}^{*}\right)(1)^{b o t} S_{r}\left(e_{j}, e_{1}+e_{j}\right) E\left(e_{j}^{*}\right)(1)^{t o p} \\
& =E\left(e_{j}^{*}\right)(1) E\left(e_{j}^{*}\right)(-1)^{t o p} S_{r}\left(e_{j}, e_{1}+e_{j}\right) E\left(e_{j}^{*}\right)(-1)^{b o t} E\left(e_{j}^{*}\right)(1) \\
& =E\left(e_{j}^{*}\right)(1) S_{r}\left(e_{1}+e_{j}, e_{1}\right) E\left(e_{j}^{*}\right)(1) \\
& =E\left(e_{j}\right)(1) S_{r}\left(e_{1}, e_{1}+e_{j}\right) E\left(e_{j}\right)(1) .
\end{aligned}
$$

By Lemma 3.6, $S_{r}\left(e_{j}+\lambda e_{i}, e_{j}\right)=S_{r}\left(e_{j} E_{j i}(\lambda), e_{j} E_{i j}(-\lambda)\right)$ is a conjugate of $S_{r}\left(e_{j}, e_{j}\right)$ by means of above type of generators.

Lemma 5.2. Let $R$ be a commutative ring in which 2 is invertible. For $\lambda, \mu \in R$, and for all $r, 2 \leq i \neq j \leq r+1$, we have

$$
\begin{aligned}
& S_{r}\left(e_{1}, e_{1}+\lambda e_{i}+\mu e_{j}\right)=\alpha \beta \beta \alpha, \\
& S_{r}\left(e_{1}+\lambda e_{i}+\mu e_{j}, e_{1}\right)=\gamma \delta \delta \gamma, \\
& S_{r}\left(e_{1}+\lambda e_{i}, e_{1}+\mu e_{j}\right)=\gamma \beta \beta \gamma,
\end{aligned}
$$

where $\alpha=S_{r}\left(e_{1}, e_{1}+\frac{\lambda}{2} e_{i}\right), \beta=S_{r}\left(e_{1}, e_{1}+\frac{\mu}{2} e_{j}\right), \gamma=S_{r}\left(e_{1}+\frac{\lambda}{2} e_{i}, e_{1}\right), \delta=$ $S_{r}\left(e_{1}+\frac{\mu}{2} e_{j}, e_{1}\right)$.

Proof. This follows from Lemma 3.2, or by a direct computation.

Corollary 5.3. Let $R$ be a commutative ring in which 2 is invertible. For $\lambda, \mu \in R$, $2 \leq i \neq j \leq r+1, r \geq 2$ and $c=e_{i}$ or $e_{i}^{*}, d=e_{j}$ or $e_{j}^{*}$, one has

$$
\left[E(c)(\mu), E(d)(\lambda)^{t b}\right]=\left[E(c)\left(\frac{\mu}{2}\right), E(d)(\lambda)\right] .
$$

Proof. We prove one case; the other cases can be proved similarly. Via Lemma 3.2, Lemma 5.2,

$$
\begin{aligned}
{\left[E\left(e_{j}\right)\right.} & \left.(\mu), E\left(e_{i}^{*}\right)(\lambda)^{b o t}\right] \\
& =E\left(e_{j}\right)(\mu) E\left(e_{i}^{*}\right)(\lambda)^{b o t} E\left(e_{j}\right)(-\mu) E\left(e_{i}^{*}\right)(\lambda)^{b o t^{-1}} \\
& =E\left(e_{j}\right)(\mu)\left\{E\left(e_{i}^{*}\right)(\lambda)^{b o t} E\left(e_{j}\right)(-\mu) E\left(e_{i}^{*}\right)(\lambda)^{t o p}\right\} E\left(e_{i}^{*}\right)(-\lambda) \\
& =E\left(e_{j}\right)(\mu) S_{r}\left(e_{1}-\mu e_{j}, e_{1}+\lambda e_{i}\right) E\left(e_{i}^{*}\right)(-\lambda) \\
& =E\left(e_{j}\right)(\mu) E\left(e_{j}\right)\left(-\frac{\mu}{2}\right) E\left(e_{i}^{*}\right)(\lambda) E\left(e_{j}\right)\left(-\frac{\mu}{2}\right) E\left(e_{i}^{*}\right)(-\lambda) \\
& =\left[E\left(e_{j}\right)\left(\frac{\mu}{2}\right), E\left(e_{i}^{*}\right)(\lambda)\right] .
\end{aligned}
$$

Definition. For $2 \leq i \leq r+1, \lambda \in R$, let

$$
\begin{aligned}
& E_{r}\left(e_{i 1}\right)(\lambda)=S_{r}\left(e_{i}+\lambda e_{1}, e_{i}\right), \\
& E_{r}\left(e_{i 1}^{*}\right)(\lambda)=S_{r}\left(e_{i}, e_{i}+\lambda e_{1}\right) .
\end{aligned}
$$

(When the size is clear, the size subscript may be dropped.)

Remark. It is easy to verify that the generators $E(c)(\lambda) S_{r}\left(e_{i}, e_{i}\right)^{-1}, c=e_{i 1}$ or $e_{i 1}^{*}$, $\lambda \in R$, satisfy the splitting property

$$
E(c)(\lambda+\mu) S_{r}\left(e_{i}, e_{i}\right)^{-1}=E(c)(\lambda) S_{r}\left(e_{i}, e_{i}\right)^{-1} \cdot E(c)(\mu) S_{r}\left(e_{i}, e_{i}\right)^{-1} .
$$


Lemma 5.4. Let $R$ be a commutative ring in which 2 is invertible. For $2 \leq i \neq$ $j \leq r+1, \lambda, \mu \in R, S_{r}\left(e_{1}+\lambda e_{j},(1-\lambda \mu) e_{1}+\mu e_{j}\right)$ can be written as a product of generators of type $E_{r}(c)(a)$, for some $a \in R, c=e_{i}$ or $e_{i}^{*}, e_{i 1}$ or $e_{i 1}^{*}$.

Proof. By Lemma 3.2, one can check that

$$
S_{r}\left((1+\lambda) e_{1}-\lambda e_{i}, e_{1}+e_{i}\right)=E\left(e_{i}\right)(-1) E\left(e_{i 1}\right)(\lambda) E\left(e_{i}\right)(-1) .
$$

(The above is a special case. For instance, the case when $\mu=-\lambda, \lambda=1$, is the transpose inverse of this case.) In general, by Lemma 3.2,

$$
\begin{aligned}
& S_{r}\left(e_{1}+\lambda e_{j},(1-\lambda \mu) e_{1}+\mu e_{j}\right) \\
& \quad=E\left(e_{j}\right)(\lambda)^{b o t} E\left(e_{j}^{*}\right)(\mu) E\left(e_{j}\right)(\lambda)^{t o p} \\
& \quad=E\left(e_{j}\right)(\lambda)^{b o t}[x,[y, z]]\left[E\left(e_{j}^{*}\right)\left(\frac{\mu}{2}\right), E\left(e_{k}\right)\left(\frac{\mu}{2}\right)\right] E\left(e_{j}\right)(\lambda)^{t o p}
\end{aligned}
$$

by biduality, where $x=E\left(e_{k}\right)\left(\frac{\mu}{2}\right), y=E\left(e_{j}^{*}\right)(1), z=E\left(e_{k}^{*}\right)(1)$, for some $k \neq j$.

The three cases which occur are similar to

$$
\begin{aligned}
& E\left(e_{j}\right)(\lambda)^{b} E\left(e_{k}\right)(\mu) E\left(e_{j}\right)(\lambda)^{t}, \\
& E\left(e_{j}\right)(\lambda)^{b} E\left(e_{j}^{*}\right)(1) E\left(e_{j}\right)(\lambda)^{t}, \\
& E\left(e_{j}\right)(\lambda)^{b} E\left(e_{k}^{*}\right)(1) E\left(e_{j}\right)(\lambda)^{t},
\end{aligned}
$$

and are easily covered by applying Lemma 3.2, Corollary 5.3, and the above special case.

Lemma 5.5. Let $R$ be a commutative ring with 1 . For $2 \leq i \neq j \leq r+1$,

(i) $\left[\left[E_{r}\left(e_{i}^{*}\right)(x), E_{r}\left(e_{j}^{*}\right)(y)\right], E_{r}\left(e_{j}\right)(z)^{t o p}\right]=E_{r}\left(e_{i}^{*}\right)(-2 x y z)^{t o p}$.

(ii) $\left[\left[E_{r}\left(e_{i}\right)(x), E_{r}\left(e_{j}\right)(y)\right], E_{r}\left(e_{j}^{*}\right)(z)^{t o p}\right]=E_{r}\left(e_{i}\right)(-2 x y z)^{t o p}$.

(iii) $\left[\left[E_{r}\left(e_{i}\right)(x), E_{r}\left(e_{j}^{*}\right)(y)\right], E_{r}\left(e_{j}\right)(z)^{t o p}\right]=E_{r}\left(e_{i}\right)(-2 x y z)^{t o p}$.

(iv) $\left[\left[E_{r}\left(e_{i}^{*}\right)(x), E_{r}\left(e_{j}\right)(y)\right], E_{r}\left(e_{j}^{*}\right)(z)^{t o p}\right]=E_{r}\left(e_{i}^{*}\right)(-2 x y z)^{t o p}$.

(v) $\left[E_{r}\left(e_{j}\right)(-z)^{b o t},\left[E_{r}\left(e_{i}^{*}\right)(x), E_{r}\left(e_{j}^{*}\right)(y)\right]\right]=E_{r}\left(e_{i}^{*}\right)(-2 x y z)^{b o t}$.

(vi) $\left[E_{r}\left(e_{j}\right)(-z)^{b o t},\left[E_{r}\left(e_{i}^{*}\right)(x), E_{r}\left(e_{j}\right)(y)\right]\right]=E_{r}\left(e_{i}\right)(-2 x y z)^{b o t}$.

(vii) $\left[E_{r}\left(e_{j}^{*}\right)(-z)^{b o t},\left[E_{r}\left(e_{i}\right)(x), E_{r}\left(e_{j}\right)(y)\right]\right]=E_{r}\left(e_{i}\right)(-2 x y z)^{b o t}$.

(viii) $\left[E_{r}\left(e_{j}^{*}\right)(-z)^{b o t},\left[E_{r}\left(e_{i}\right)(x), E_{r}\left(e_{j}^{*}\right)(y)\right]\right]=E_{r}\left(e_{i}^{*}\right)(-2 x y z)^{b o t}$.

Proof. We prove (i); the rest are proved similarly. First we consider the case when $i=2$. By Lemma 4.1,

$$
\left[E\left(e_{2}^{*}\right)(x), E\left(e_{j}^{*}\right)(y)\right]=\left(\begin{array}{cc}
E\left(e_{j-1}^{*}\right)(2 x y)^{b o t} & 0 \\
0 & E\left(e_{j-1}^{*}\right)(-2 x y)^{t o p}
\end{array}\right) .
$$

Hence for $P=E\left(e_{j-1}^{*}\right)(2 x y)^{b o t}$ and $Q=E\left(e_{j-1}^{*}\right)(-2 x y)^{t o p}$, we have

$$
\begin{gathered}
{\left[\left[E\left(e_{2}^{*}\right)(x), E\left(e_{j}^{*}\right)(y)\right], E\left(e_{j}\right)(z)^{t o p}\right]} \\
\quad=\left(\begin{array}{cc}
P & 0 \\
0 & Q
\end{array}\right)\left(\begin{array}{cc}
I & S_{r-1}\left(z e_{j-1}, 0\right) \\
0 & I
\end{array}\right)\left(\begin{array}{cc}
P^{-1} & 0 \\
0 & Q^{-1}
\end{array}\right)\left(\begin{array}{cc}
I & -S_{r-1}\left(z e_{j-1}, 0\right) \\
0 & I
\end{array}\right) \\
\quad=\left(\begin{array}{cc}
I & -S_{r-1}\left(z e_{j-1}, 0\right)+P S_{r-1}\left(z e_{j-1}, 0\right) Q^{-1} \\
0 & I
\end{array}\right) .
\end{gathered}
$$


Now

$$
\begin{aligned}
& -S_{r-1}\left(z e_{j-1}, 0\right)+P S_{r-1}\left(z e_{j-1}, 0\right) Q^{-1} \\
& \quad=-S_{r-1}\left(z e_{j-1}, 0\right)+\left(\begin{array}{cc}
0 & S_{r-2}\left(z e_{j-2}, 0\right) \\
-S_{r-2}\left(0, z e_{j-2}\right)^{T} & -2 x y z I_{2^{r-2}}
\end{array}\right) \\
& \quad=\left(\begin{array}{cc}
0 & 0 \\
0 & -2 x y z I_{2^{r-2}}
\end{array}\right)=S_{r-1}\left(0,-2 x y z e_{1}\right) .
\end{aligned}
$$

Thus

$$
\begin{aligned}
{\left[\left[E\left(e_{2}^{*}\right)(x), E\left(e_{j}^{*}\right)(y)\right], E\left(e_{j}\right)(z)^{t o p}\right] } & =\left(\begin{array}{cc}
I_{2^{r-1}} & S_{r-1}\left(0,-2 x y z e_{1}\right) \\
0 & I_{2^{r-1}}
\end{array}\right) \\
& =E\left(e_{2}^{*}\right)(-2 x y z)^{t o p}
\end{aligned}
$$

as required. We now consider the case when $j=2$. By Lemma 4.1,

$$
\left[E\left(e_{i}^{*}\right)(x), E\left(e_{2}^{*}\right)(y)\right]=\left(\begin{array}{cc}
E\left(e_{i-1}^{*}\right)(-2 x y)^{b o t} & 0 \\
0 & E\left(e_{i-1}^{*}\right)(2 x y)^{t o p}
\end{array}\right) .
$$

Hence for $P=E\left(e_{i-1}^{*}\right)(-2 x y)^{b o t}$ and $Q=E\left(e_{i-1}^{*}\right)(2 x y)^{t o p}$, we have

$$
\begin{gathered}
{\left[\left[E\left(e_{i}^{*}\right)(x), E\left(e_{2}^{*}\right)(y)\right], E\left(e_{2}\right)(z)^{t o p}\right]} \\
\quad=\left(\begin{array}{cc}
P & 0 \\
0 & Q
\end{array}\right)\left(\begin{array}{cc}
I & S_{r-1}\left(z e_{1}, 0\right) \\
0 & I
\end{array}\right)\left(\begin{array}{cc}
P^{-1} & 0 \\
0 & Q^{-1}
\end{array}\right)\left(\begin{array}{cc}
I & -S_{r-1}\left(z e_{1}, 0\right) \\
0 & I
\end{array}\right) \\
\quad=\left(\begin{array}{cc}
I & -S_{r-1}\left(z e_{1}, 0\right)+P S_{r-1}\left(z e_{1}, 0\right) Q^{-1} \\
0 & I
\end{array}\right) .
\end{gathered}
$$

Now

$$
\begin{aligned}
& -S_{r-1}\left(z e_{1}, 0\right)+P S_{r-1}\left(z e_{1}, 0\right) Q^{-1} \\
& \quad=-S_{r-1}\left(z e_{1}, 0\right)+\left(\begin{array}{cc}
z I_{2^{r-2}} & -S_{r-2}\left(0,2 x y z e_{i-2}\right) \\
S_{r-2}\left(2 x y z e_{i-2}, 0\right)^{T} & 0
\end{array}\right) \\
& \quad=\left(\begin{array}{cc}
0 & -S_{r-2}\left(0,2 x y z e_{i-2}\right) \\
S_{r-2}\left(2 x y z e_{i-2}, 0\right)^{T} & 0
\end{array}\right)=S_{r-1}\left(0,-2 x y z e_{i-1}\right) .
\end{aligned}
$$

Thus

$$
\begin{aligned}
{\left[\left[E\left(e_{i}^{*}\right)(x), E\left(e_{2}^{*}\right)(y)\right], E\left(e_{2}\right)(z)^{t o p}\right] } & =\left(\begin{array}{cc}
I_{2^{r-1}} & S_{r-1}\left(0,-2 x y z e_{i-1}\right) \\
0 & I_{2^{r-1}}
\end{array}\right) \\
& =E\left(e_{i}^{*}\right)(-2 x y z)^{t o p},
\end{aligned}
$$

as required.

Now we consider the general the case when $2<i \neq j \leq r+1$. As noted in the proof of Lemma 4.1,

$$
\left[E_{r}\left(e_{i}^{*}\right)(x), E_{r}\left(e_{j}^{*}\right)(y)\right]=\left(\begin{array}{cc}
A & 0 \\
0 & A
\end{array}\right)
$$


where $A=I-2 S_{r-1}\left(0, x e_{i-1}\right) S_{r-1}\left(y e_{j-1}, 0\right)^{T}$. Hence,

$$
\begin{aligned}
{\left[\left[E_{r}\left(e_{i}^{*}\right)(x), E_{r}\left(e_{j}^{*}\right)(y)\right], E_{r}\left(e_{j}\right)^{t o p}(z)\right] } & \\
= & \left(\begin{array}{cc}
A & 0 \\
0 & A
\end{array}\right)\left(\begin{array}{cc}
I & S_{r-1}\left(z e_{j-1}, 0\right) \\
0 & I
\end{array}\right)\left(\begin{array}{cc}
B & 0 \\
0 & B
\end{array}\right)\left(\begin{array}{cc}
I & -S_{r-1}\left(z e_{j-1}, 0\right) \\
0 & I
\end{array}\right) \\
\quad= & \left(\begin{array}{cc}
A & A S_{r-1}\left(z e_{j-1}, 0\right) \\
0 & A
\end{array}\right)\left(\begin{array}{cc}
B & -B S_{r-1}\left(z e_{j-1}, 0\right) \\
0 & B
\end{array}\right) \\
= & \left(\begin{array}{cc}
I & -S_{r-1}\left(z e_{j-1}, 0\right)+A S_{r-1}\left(z e_{j-1}, 0\right) B \\
0 & I
\end{array}\right),
\end{aligned}
$$

for $B=I+2 S_{r-1}\left(0, x e_{i-1}\right) S_{r-1}\left(y e_{j-1}, 0\right)^{T}$. As in the proof of Lemma 4.3,

$$
\begin{aligned}
-S_{r-1} & \left(z e_{j-1}, 0\right)+A S_{r-1}\left(z e_{j-1}, 0\right) B \\
& =-S_{r-1}\left(z e_{j-1}, 0\right)+A\left\{B S_{r-1}\left(z e_{j-1}, 0\right)-2 y z S_{r-1}\left(0, x e_{i-1}\right)\right\} \\
& =A S_{r-1}\left(0,-2 x y z e_{i-1}\right) \\
& =\left\{I-2 S_{r-1}\left(0, x e_{i-1}\right) S_{r-1}\left(y e_{j-1}, 0\right)^{T}\right\} S_{r-1}\left(0,-2 x y z e_{i-1}\right) \\
& =S_{r-1}\left(0,-2 x y z e_{i-1}\right) .
\end{aligned}
$$

Hence

$$
\begin{aligned}
{\left[\left[E_{r}\left(e_{i}^{*}\right)(x), E_{r}\left(e_{j}^{*}\right)(y)\right], E_{r}\left(e_{j}\right)^{t o p}(z)\right] } & =\left(\begin{array}{cc}
I & S_{r-1}\left(0,-2 x y z e_{i-1}\right) \\
0 & I
\end{array}\right) \\
& =E_{r}\left(e_{i}^{*}\right)(-2 x y z)^{t o p}
\end{aligned}
$$

as required.

Proposition 5.6. Let $R$ be a commutative ring in which 2 is invertible. Then $E U m_{r}(R)^{t b}=E U m_{r}(R)$.

Proof. $\operatorname{EUm}_{r}(R)$ is generated by the Suslin matrices $S_{r}(v, w)$, with $v \in e_{1} E_{r+1}(R)$, and with $w$ such that $\langle v, w\rangle=1$. As before, there exists $\varepsilon_{1} \in E_{r+1}(R)$ such that $v_{1}=e_{1} \varepsilon_{1}, w_{1}=e_{1} \varepsilon_{1}^{T^{-1}}$. Therefore, by our key lemma, Lemma 3.2, $E U m_{r}(R) \subset$ $E U m_{r}(R)^{t b}$. (This part does not need the fact that 2 is invertible in $R$.)

For the converse part, since 2 is invertible in $R$, it suffices to show that $E_{r}\left(e_{i}^{*}\right)(-2 x y z)^{t o p}, E_{r}\left(e_{i}^{*}\right)(-2 x y z)^{b o t} \in E U m_{r}(R)$. By Lemma 5.5, we have

$$
\begin{aligned}
E_{r}\left(e_{i}^{*}\right)(-2 x y z)^{t o p}= & {\left[\left[E_{r}\left(e_{i}^{*}\right)(x), E_{r}\left(e_{j}^{*}\right)(y)\right], E_{r}\left(e_{j}\right)(z)^{t o p}\right] } \\
= & \left\{\left[E_{r}\left(e_{i}^{*}\right)(x), E_{r}\left(e_{j}^{*}\right)(y)\right]\right\} \\
& \left\{E_{r}\left(e_{j}\right)(z)^{t o p} E_{r}\left(e_{j}^{*}\right)(y) E_{r}\left(e_{j}\right)(z)^{b o t}\right\}\left\{E_{r}\left(e_{j}\right)(z)^{-1}\right\} \\
& \left\{E_{r}\left(e_{j}\right)(z)^{t o p} E_{r}\left(e_{i}^{*}\right)(x) E_{r}\left(e_{j}\right)(z)^{b o t}\right\}\left\{E_{r}\left(e_{j}\right)(z)^{-1}\right\} \\
& \left\{E_{r}\left(e_{j}\right)(z)^{t o p} E_{r}\left(e_{j}^{*}\right)(y)^{-1} E_{r}\left(e_{j}\right)(z)^{b o t}\right\}\left\{E_{r}\left(e_{j}\right)(z)^{-1}\right\} \\
& \left\{E_{r}\left(e_{j}\right)(z)^{t o p} E_{r}\left(e_{i}^{*}\right)(x)^{-1} E_{r}\left(e_{j}\right)(z)^{b o t}\right\}\left\{E_{r}\left(e_{j}\right)(z)^{-1}\right\} .
\end{aligned}
$$

By Lemma 3.2 and Lemma 5.2,

$$
E_{r}\left(e_{j}\right)(z)^{t o p} E_{r}\left(e_{i}^{*}\right)(x) E_{r}\left(e_{j}\right)(z)^{b o t}=S_{r}\left(e_{1}+z e_{j}, e_{1}+x e_{i}\right)=\alpha \beta \beta \alpha,
$$

where $\alpha=E_{r}\left(e_{j}\right)\left(\frac{z}{2}\right)$ and $\beta=E_{r}\left(e_{i}^{*}\right)\left(\frac{x}{2}\right)$. Again by Lemma 3.2 and Lemma 5.4, we have

$$
E_{r}\left(e_{j}\right)(z)^{t o p} E_{r}\left(e_{j}^{*}\right)(y) E_{r}\left(e_{j}\right)(z)^{b o t}=S_{r}\left((1-y z) e_{1}+z e_{j}, e_{1}+y e_{j}\right) \in E U m_{r}(R) .
$$


Therefore,

$$
\begin{aligned}
E\left(e_{i}^{*}\right) & (-2 x y z)^{\text {top }} \\
= & \left\{E\left(e_{i}^{*}\right)(x)\right\}\left\{E\left(e_{j}^{*}\right)(y)\right\}\left\{E\left(e_{i}^{*}\right)(x)^{-1}\right\}\left\{E\left(e_{j}^{*}\right)(y)^{-1}\right\} \\
& \left\{S_{r}\left((1-y z) e_{1}+z e_{j}, e_{1}+y e_{j}\right)\right\}\left\{E\left(e_{j}\right)(z)^{-1}\right\} \\
& \left\{E\left(e_{j}\right)\left(\frac{z}{2}\right)\right\}\left\{E\left(e_{i}^{*}\right)\left(\frac{x}{2}\right)\right\}\left\{E\left(e_{i}^{*}\right)\left(\frac{x}{2}\right)\right\}\left\{E\left(e_{j}\right)\left(\frac{z}{2}\right)\right\} \\
& \left\{E\left(e_{j}\right)(z)^{-1}\right\}\left\{S_{r}\left((1+y z) e_{1}+z e_{j}, e_{1}-y e_{j}\right)\right\}\left\{E\left(e_{j}\right)(z)^{-1}\right\} \\
& \left\{E\left(e_{j}\right)\left(\frac{z}{2}\right)\right\}\left\{E\left(e_{i}^{*}\right)\left(\frac{x}{2}\right)^{-1}\right\}\left\{E\left(e_{i}^{*}\right)\left(\frac{x}{2}\right)^{-1}\right\}\left\{E\left(e_{j}\right)\left(\frac{z}{2}\right)\right\}\left\{E\left(e_{j}\right)(z)^{-1}\right\} .
\end{aligned}
$$

Hence, $E_{r}\left(e_{i}^{*}\right)(-2 x y z)^{t o p} \in E U m_{r}(R)$.

We now show that "reversing above product" gives $E_{r}\left(e_{i}^{*}\right)(-2 x y z)^{b o t}$ :

$$
\begin{aligned}
& \left\{E_{r}\left(e_{j}\right)(z)^{-1}\right\}\left\{E_{r}\left(e_{j}\right)\left(\frac{z}{2}\right)\right\}\left\{E_{r}\left(e_{i}^{*}\right)\left(\frac{x}{2}\right)^{-1}\right\}\left\{E_{r}\left(e_{i}^{*}\right)\left(\frac{x}{2}\right)^{-1}\right\}\left\{E_{r}\left(e_{j}\right)\left(\frac{z}{2}\right)\right\} \\
& \left\{E_{r}\left(e_{j}\right)(z)^{-1}\right\}\left\{S_{r}\left((1+y z) e_{1}+z e_{j}, e_{1}-y e_{j}\right)\right\} \\
& \left\{E_{r}\left(e_{j}\right)(z)^{-1}\right\}\left\{E_{r}\left(e_{j}\right)\left(\frac{z}{2}\right)\right\}\left\{E_{r}\left(e_{i}^{*}\right)\left(\frac{x}{2}\right)\right\}\left\{E_{r}\left(e_{i}^{*}\right)\left(\frac{x}{2}\right)\right\}\left\{E_{r}\left(e_{j}\right)\left(\frac{z}{2}\right)\right\} \\
& \left\{E_{r}\left(e_{j}\right)(z)^{-1}\right\}\left\{S_{r}\left((1-y z) e_{1}+z e_{j}, e_{1}+y e_{j}\right)\right\} \\
& \left\{E_{r}\left(e_{j}^{*}\right)(y)^{-1}\right\}\left\{E_{r}\left(e_{i}^{*}\right)(x)^{-1}\right\}\left\{E_{r}\left(e_{j}^{*}\right)(y)\right\}\left\{E_{r}\left(e_{i}^{*}\right)(x)\right\} \\
= & \left\{E_{r}\left(e_{j}\right)(z)^{-1}\right\}\left\{E_{r}\left(e_{j}\right)(z)^{t} E_{r}\left(e_{i}^{*}\right)(x)^{-1} E_{r}\left(e_{j}\right)(z)^{b}\right\} \\
& \left\{E_{r}\left(e_{j}\right)(z)^{-1}\right\}\left\{E_{r}\left(e_{j}\right)(z)^{t} E_{r}\left(e_{j}^{*}\right)(y)^{-1} E_{r}\left(e_{j}\right)(z)^{b}\right\}\left\{E_{r}\left(e_{j}\right)(z)^{-1}\right\} \\
& \left\{E_{r}\left(e_{j}\right)(z)^{t} E_{r}\left(e_{i}^{*}\right)(x) E_{r}\left(e_{j}\right)(z)^{b}\right\}\left\{E_{r}\left(e_{j}\right)(z)^{-1}\right\} \\
& \left\{E_{r}\left(e_{j}\right)(z)^{t} E_{r}\left(e_{j}^{*}\right)(y) E_{r}\left(e_{j}\right)(z)^{b}\right\}\left\{\left[E_{r}\left(e_{i}^{*}\right)(x), E_{r}\left(e_{j}^{*}\right)(y)\right]^{-1}\right\} \\
= & E_{r}\left(e_{j}\right)(-z)^{b}\left[E_{r}\left(e_{i}^{*}\right)(x)^{-1}, E_{r}\left(e_{j}^{*}\right)(y)^{-1}\right] E_{r}\left(e_{j}\right)(z)^{b}\left[E_{r}\left(e_{i}^{*}\right)(x), E_{r}\left(e_{j}^{*}\right)(y)\right]^{-1} \\
= & E_{r}\left(e_{j}\right)(-z)^{b}\left[E_{r}\left(e_{i}^{*}\right)(-x), E_{r}\left(e_{j}^{*}\right)(-y)\right] E_{r}\left(e_{j}\right)(z)^{b}\left[E_{r}\left(e_{i}^{*}\right)(x), E_{r}\left(e_{j}^{*}\right)(y)\right]^{-1} \\
= & E_{r}\left(e_{j}\right)(-z)^{b}\left[E_{r}\left(e_{j}^{*}\right)(y), E_{r}\left(e_{i}^{*}\right)(x)\right] E_{r}\left(e_{j}\right)(z)^{b}\left[E_{r}\left(e_{i}^{*}\right)(x), E_{r}\left(e_{j}^{*}\right)(y)\right]^{-1} \\
= & {\left[E_{r}\left(e_{j}\right)(-z)^{b},\left[E_{r}\left(e_{i}^{*}\right)(x), E_{r}\left(e_{j}^{*}\right)(y)\right]\right]=E_{r}\left(e_{i}^{*}\right)(-2 x y z)^{b} . }
\end{aligned}
$$

Theorem 5.7. Let $R$ be a commutative ring in which 2 is invertible. Let $\varepsilon \in$ $E_{r+1}(R)$. Then $S_{r}\left(v \varepsilon, w \varepsilon^{T^{-1}}\right) \in H$, where $H$ is the subgroup of $E U m_{r}(R)$ generated by elements of the type $E_{r}(c)(\lambda)$, for $c=e_{i}, e_{i}^{*}, e_{i 1}$, or $e_{i 1}^{*}$. In fact, $S_{r}\left(v \varepsilon, w \varepsilon^{T^{-1}}\right)=\alpha S_{r}(v, w) \alpha^{*}$, where $\alpha=S_{1} S_{2} \cdots S_{n}, \alpha^{*}=S_{n} S_{n-1} \cdots S_{1}$, where each $S_{i}$ is of the type $E_{r}(c)(\lambda)$, for $c=e_{i}, e_{i}^{*}, e_{i 1}$, or $e_{i 1}^{*}$

Proof. By our key lemma, one can find $\alpha \in E U m_{r}(R)^{t b}, \alpha^{*} \in E U m_{r}(R)^{b t}$ (obtained by reversing the order and replacing top by bottom) such that $S_{r}\left(v \varepsilon, w \varepsilon^{T^{-1}}\right)$ $=\alpha S_{r}(v, w) \alpha^{*}$. Now apply Proposition 5.6. The proof of Proposition 5.6 gives a procedure of how we can write $\alpha$ as a product of required type above, so that $\alpha^{*}$ is obtained by reversing the order of the product describing $\alpha$.

In view of the above lemma one gets the structure theorem for the Elementary Unimodular vector group $E U m_{r}(R)$, when 2 is invertible in $R$ :

Theorem 5.8. Let $R$ be a commutative ring in which 2 is invertible. Then $E U m_{r}(R)$ is generated by elements of the form $E\left(e_{i}\right)(w), E\left(e_{i}^{*}\right)(x), E\left(e_{i 1}\right)(y)$, $E\left(e_{i 1}^{*}\right)(z)$, for $w, x, y, z \in R, 2 \leq i \leq r+1$. 
Remark 5.9. One can show that $\operatorname{EUm}_{r}(R)=E U m_{r}(R)^{t b}$, for any commutative ring $R$, without any additional assumption (cf. [1, Proposition 2.6]). However, it is not clear to us, when $2 R \neq R$, whether the set of generators mentioned in Theorem 5.8 will suffice to generate $E U m_{r}(R)$.

\section{ACKNOWLEDGEMENTS}

We thank the referee for input which led to improvements in the text, and also for correcting several technical details. The referee also made several other incisive remarks, especially on how one should view Corollary 3.3. We shall elaborate on this viewpoint in an article titled 'A Fundamental property of Suslin Matrices'.

\section{REFERENCES}

[1] Jose, S., Rao R. A., A local global principle for the elementary unimodular vector group, Commutative Algebra and Algebraic Geometry (Bangalore, India, 2003), Contemp. Math., vol. 390, Amer. Math. Soc., Providence, RI, 2005, pp. 119-125.

[2] Rao, R. A., Jose, S., A fundamental property of Suslin matrices, in preparation.

[3] Suslin, A. A., Vaserstein, L. N., Serre's problem on projective modules over polynomial rings and algebraic K-theory, Math. USSR Izvestija 10 (1976), 937-1001. MR0447245 (56:5560)

[4] Suslin, A. A., On stably free modules, Math. USSR Sbornik 31 (1977), 479-491. MR0441949 $(56: 340)$

[5] Suslin, A. A., On the structure of the special linear group over polynomial rings, Math. USSR Izvestija 11 (1977), 221-238. MR0472792 (57:12482)

Department of Mathematics, Ismail Yusuf College, Jogeshwari(E), Mumbai 400-060, INDIA

E-mail address: selbyjose@rediffmail.com

School of Mathematics, Tata Institute of Fundamental Research, Dr. Homi Bhabha RoAd, Mumbai 400 005, India

E-mail address: ravi@math.tifr.res.in 